\title{
A numerical mode matching method for wave scattering in a layered medium with a stratified inhomogeneity
}

\author{
Wangtao $\mathrm{Lu}^{*} \quad$ Ya Yan $\mathrm{Lu}^{\dagger} \quad$ Dawei Song ${ }^{\ddagger}$
}

April 24, 2018

\begin{abstract}
Numerical mode matching (NMM) methods are widely used for analyzing wave propagation and scattering in structures that are piecewise uniform along one spatial direction. For open structures that are unbounded in transverse directions (perpendicular to the uniform direction), the NMM methods use the perfectly matched layer (PML) technique to truncate the transverse variables. When incident waves are specified in homogeneous media surrounding the main structure, the total field is not always outgoing, and the NMM methods rely on reference solutions for each uniform segment. Existing NMM methods have difficulty handing gracing incident waves and special incident waves related to the onset of total internal reflection, and are not very efficient at computing reference solutions for non-plane incident waves. In this paper, a new NMM method is developed to overcome these limitations. A Robin-type boundary condition is proposed to ensure that non-propagating and non-decaying wave field components are not reflected by truncated PMLs. Exponential convergence of the PML solutions based on the hybrid Dirichlet-Robin boundary condition is established theoretically. A fast method is developed for computing reference solutions for cylindrical incident waves. The new NMM
\end{abstract}

*School of Mathematical Sciences, Zhejiang University, Hangzhou 310027, China. Email: wangtaolu@zju.edu.cn (corresponding author).

${ }^{\dagger}$ Department of Mathematics, City University of Hong Kong, Kowloon, Hong Kong, China. Email: mayylu@cityu.edu.hk.

${ }^{\ddagger}$ Department of Mathematics, Nanjing University of Aeronautics and Astronautics, Nanjing, Jiangsu, China. Email: dwsmath@nuaa.edu.cn. 
is implemented for two-dimensional structures and polarized electromagnetic waves. Numerical experiments are carried out to validate the new NMM method and to demonstrate its performance.

\section{Introduction}

Wave scattering problems in a layered medium with a penetrable or impenetrable inhomogeneity appear in numerous scientific and engineering applications [9]. Classical numerical methods such as the finite difference method, the finite element method (FEM) [23], and the spectral method are very versatile, but are not always the most efficient, since they need to discretize the whole computational domain. For piecewise homogeneous structures, the boundary integral equation (BIE) methods [7, 6, 17, 21] are highly competitive since they discretize only the interfaces and the boundary of the inhomogeneity. If the structure can be divided into a number of segments or regions where the governing equation becomes separable, the mode matching method, a.k.a mode expansion method or modal method [5, 19, 26], and its many numerical variants [10, 12, 13, 16, 18, 20, 22, 24, 29, 11, 3, 4] may be used. Typically, these methods are applicable if the structure is piecewise uniform along one spatial direction. In each uniform segment, the wave field is expanded in eigenmodes of a related transverse differential operator, and the expansion coefficients are solved from a linear system obtained by matching the wave field at the interfaces between neighboring segments. The classical mode matching method solves the eigenmodes analytically. The numerical mode matching (NMM) methods solve the eigenmodes by numerical methods, and they are easier to implement and applicable to more general structures. The mode matching method and its variants have the advantage of avoiding discretizing one spatial variable. They are widely used in engineering applications, since many designed structures are indeed piecewise uniform.

For numerical simulations of waves, the perfectly matched layer (PML) 2] is an important technique for truncating unbounded domains. It is widely used with standard numerical methods, such as FEM, that discretize the whole computational domain. The BIE methods usually automatically take care of the radiation conditions at infinity, but for scattering problems in layerd media, PML can also be used to efficiently truncate interfaces that extend to infinity [21]. For NMM methods, PML was first applied to study piecewise uniform waveguides [11, 3, 4]. An optical waveguide is an open structure, i.e., the transverse domain perpendicular to the waveguide axis is unbounded. Analytic mode matching method is difficult to use, since the 
transverse operator has a continuous spectrum and field expansions contain integrals related to the radiation modes. When a PML is used to truncate the transverse domain, typically with a zero Dirichlet boundary condition at the external boundary of the PML, the continuous spectrum is discretized, and the field expansions are approximated by sums of discrete eigenmodes.

For many applications, an incident wave is specified in the homogeneous media surrounding the scatterer, then the total wave field in each uniform segment does not satisfy outgoing radiation conditions in the transverse directions, and is incompatible with the eigenmodes computed using a PML. To overcome this difficulty, we can find a reference solution for the given incident wave in each uniform segment, and then expand the difference between the total field and the reference solution in the PML-based eigenmodes [22]. Typically, the field difference in each segment is indeed outgoing in the transverse directions, and a NMM method based on this approach works reasonably well. But unfortunately, the method breaks down in special circumstances where the field difference in a segment contains a component that is exactly or nearly invariant in the transverse direction, i.e., a component with a zero transverse wavenumber. This happens if the incident wave has the critical incident angle for the onset of total internal reflection in the exterior segments. In that case, the field difference in any interior segment contains a non-propagating and non-decaying component with a zero or near zero transverse wavenumber. This difficulty also arises when the incident wave is nearly parallel to the uniform direction, i.e., a gracing incidence. In that case, the field difference in an interior segment also contains a plane wave component with a near zero transverse wavenumber.

In this paper, we develop a new NMM method to overcome the above difficulty. Our approach is to use a Robin boundary condition for the PML in the interior segments. The boundary condition is designed to ensure that the field component with a zero or near zero transverse wavenumber is not reflected by the PML. A similar Robin-type condition for PML was previously used by one of the authors to preserve a weakly confined guided mode propagating in optical waveguides [14]. For the exterior segments, we keep the simple zero Dirichlet boundary condition. To give the method a theoretical foundation, we analyze the effectiveness of the PML using hybrid Dirichlet-Robin boundary conditions. It is shown that the error induced by the PML decays exponentially with the thickness or the absorbing coefficient of the PML. For scattering problems with incident waves from a point or line source, the NMM method faces an additional difficulty, namely, the computation of the reference solutions, especially for the segment involving the inhomogeneity. The traditional approach that turns a point or line source to plane waves by Fourier transform is not very efficient. We develop an efficient 
method for computing the reference solutions based the PML technique and the method of separation of variables.

The rest of this paper is organized as follows. In Section 2, we formulate the scattering problem, review the PML theory. In Section 3, we describe an NMM method. In Section 4, we derive the new Robin-type boundary condition and show that the solution based on a PML and a hybrid Dirichlet-Robin condition converges to the true scattering solution exponentially. In Section 5 , we develop an efficient method for computing reference solutions when the incident wave is a line source. In Section 6, we present a few numerical examples to validate our NMM method and to illustrate its performance. The paper is concluded by some remarks and discussions in Section 7 .

\section{Problem formulation}

To simplify the presentation, we begin with a scattering problem in a twolayer medium. The physical structure is characterized by a $z$-invariant dielectric function

$$
\varepsilon(x, y)=\left\{\begin{array}{lc}
\varepsilon_{+}=n_{+}^{2}, & (x, y) \in \mathbb{R}_{+}^{2} \backslash \bar{D} \\
\tilde{\varepsilon}(y), & (x, y) \in D \\
\varepsilon_{-}=n_{-}^{2}, & (x, y) \in \mathbb{R}_{-}^{2} \backslash \bar{D}
\end{array}\right.
$$

where $\mathbb{R}_{ \pm}^{2}=\left\{(x, y) \in \mathbb{R}^{2}: \pm y>0\right\}, \tilde{\varepsilon}(y) \geq 1$ is piecewise smooth on $\left(y_{0}, y_{1}\right)$, $D$ is a rectangle $\left(-x_{0}, x_{0}\right) \times\left(y_{0}, y_{1}\right)$ with $x_{0}>0, y_{1} \geq 0$ and $y_{0} \leq 0$, and it corresponds to a stratified inhomogeneity. In $\mathbb{R}_{+}^{2} / \bar{D}$, we specify a plane incident wave $u^{\text {inc }}=e^{i\left(\alpha x-\beta_{+} y\right)}$, where $\alpha=k_{0} n_{+} \sin \theta, \beta_{+}=k_{0} n_{+} \cos \theta$, and $\theta \in(-\pi / 2, \pi / 2)$ is the incident angle. The total wave field $u^{\text {tot }}$ satisfies the Helmholtz equation

$$
\Delta u^{\text {tot }}+k_{0}^{2} \varepsilon(x, y) u^{\text {tot }}=0,
$$

where $\Delta=\partial_{x}^{2}+\partial_{y}^{2}$, and $k_{0}$ is the free-space wavenumber. Across an interface or discontinuity, we have the following transmission condition

$$
\left[u^{\text {tot }}\right]=0, \quad\left[\frac{\partial u^{\text {tot }}}{\partial \boldsymbol{\nu}}\right]=0
$$

eq: trans : cond

where $\boldsymbol{\nu}$ is the unit normal vector on the interface pointing towards $\mathbb{R}_{-}^{2}$, and $[\cdot]$ denotes the jump of the quantity across the interface. For electromagnetic waves in the $E$ polarization, $u^{\text {tot }}$ is the $z$ component (the only nonzero component) of the electric field. 
At infinity, the scattered wave field defined as

$$
u^{s}= \begin{cases}u^{\mathrm{tot}}-u_{0}^{\mathrm{tot}}, & \text { in } \mathbb{R}^{2} / \bar{D}, \\ u^{\mathrm{tot}}, & \text { in } D,\end{cases}
$$

satisfies the half-plane Sommerfeld radiation condition in both $\mathbb{R}_{+}^{2}$ and $\mathbb{R}_{-}^{2}$, i.e.,

$$
\lim _{r \rightarrow \infty} \sqrt{r}\left(\partial_{r} u^{s}-i k_{0} n_{ \pm} u^{s}\right)=0, \quad r=\sqrt{x^{2}+y^{2}}, \quad(x, y): \text { eq } \in \mathbb{R}_{ \pm}^{\text {f }}: \text { Sem }: \text { cond }
$$

Here, $u_{0}^{\text {tot }}$ is the solution for the same incident wave in the background twolayer medium without the inhomogeneity. More precisely, we have

$$
\begin{aligned}
& u_{0}^{\text {tot }}= \begin{cases}e^{i\left(\alpha x-\beta_{+} y\right)}+R e^{i\left(\alpha x+\beta_{+} y\right)} & \left.(x, y) \in \mathbb{R}_{+}^{2}, \text { eq }: \text { sol : cond } ; 5\right) \\
(R+1) e^{i\left(\alpha x-\beta_{-} y\right)} & (x, y) \in \mathbb{R}_{-}^{2},\end{cases} \\
& \beta_{-}=\sqrt{k_{0}^{2} \varepsilon_{-}-\alpha^{2}}, \quad R=\frac{\beta_{+}-\beta_{-}}{\beta_{+}+\beta_{-}}, \quad \text { eq: sol : para }
\end{aligned}
$$

According to [25, 8, 1, we have the following existence and uniqueness results:

Theorem 2.1. For any incident plane wave with $k_{0}>0$, the scattering problem (2), (3), (4) has a unique solution $u^{\text {tot }}$ in $H_{\text {loc }}^{1}\left(\mathbb{R}^{2}\right)$.

Since $u^{s}$ is outgoing, the PML technique [2] can be used to truncate $\mathbb{R}^{2}$. Let us define the following complex coordinate stretching functions

$$
\tilde{x}(x)=x+i \int_{0}^{x} \sigma_{1}(t) d t, \quad \tilde{y}(y)=y+i \int_{0}^{y} \sigma_{2}(t) d t, \quad \text { eq }: \text { pml }:(x)
$$

where $\sigma_{l}(t)=\sigma_{l}(-t)$ for all $t, \sigma_{l}(t)=0$ for $|t| \leq L_{l} / 2$, and $\sigma_{l}(t)>0$ for $|t|>L_{l} / 2$, and $L_{l}>0$ for $l=1,2$. Notice that the rectangle $B_{1}=$ $\left(-L_{1} / 2, L_{1} / 2\right) \times\left(-L_{2} / 2, L_{2} / 2\right)$ encloses the inhomogeneity $D$, and the rectangle $B_{2}=\left(-L_{1} / 2-d_{1}, L_{1} / 2+d_{1}\right) \times\left(-L_{2} / 2-d_{2}, L_{2} / 2+d_{2}\right)$ is used to truncate $\mathbb{R}^{2}$. Based on Green's representation formula, the extension of $u^{s}$ in $B_{2}$ can be defined, then

$$
\tilde{u}^{s}(x, y):=u^{s}(\tilde{x}(x), \tilde{y}(y))
$$

satisfies the following PML-Helmholtz equation

$$
\begin{array}{ll}
\nabla \cdot\left(A \nabla \tilde{u}^{s}\right)+\alpha_{1}(x) \alpha_{2}(y) k_{0}^{2} \varepsilon(x, y) \tilde{u}^{s}=0, & \text { eq:upml }(\dot{1}) \mid \\
\tilde{u}^{s}(x, 0+)=\tilde{u}^{s}(x, 0-), & \partial_{\tilde{y}} \tilde{u}^{s}(x, 0+)=\partial_{\tilde{y}} \tilde{u}^{s}(x, 0-), \text { in } \mathbb{R} /\left[-x_{0}, \text { eq }_{0}:{ }^{\text {upml }}: \dot{2}{ }^{(9}\right)
\end{array}
$$


where $A=\operatorname{diag}\left(\alpha_{2}(y) / \alpha_{1}(x), \alpha_{1}(x) / \alpha_{2}(y)\right)$, and $\alpha_{l}=1+i \sigma_{l}$. Typically, a zero Dirichlet boundary condition is enforced on $\Gamma_{2}=\partial B_{2}$, i.e.,

$$
\tilde{u}^{s}(x, y)=0, \quad \text { on } \quad \Gamma_{2} .
$$

eq: $\operatorname{upml}: 3$

The following theorem characterizes the exponential convergence of the PML solution.

Theorem 2.2. Let $\sigma_{1}=\sigma_{2} \equiv \sigma$ and $d_{1}=d_{2}=d$ in the PML for some positive constants $\sigma$ and $d$, and let $\bar{\sigma}=\sigma d$ such that $\gamma_{0} \bar{\sigma} \geq \max \left(k_{\min }^{-1}, d\right)$, where $\gamma_{0}=d / \sqrt{\left(L_{1}+d\right)^{2}+\left(L_{2}+d\right)^{2}}$. Then for sufficiently large $\bar{\sigma}$, the $P M L$ problem (810) has a unique solution $\tilde{u}^{s}$ in $H^{1}\left(B_{2}\right)$. Moreover, there exists a constant $C$, which depends only on $\gamma_{0}, k_{\max } / k_{\min }$, and $L_{2} / L_{1}$, but independent of $n_{-}, n_{+}, L_{1}, L_{2}$, and $d$, such that

$$
\begin{aligned}
\left\|u^{s}-\tilde{u}^{s}\right\|_{H^{1}\left(B_{1}\right)} & \leq C\left(1+\hat{C}^{-1}\right) \gamma_{1}\left(1+k_{\min } L_{1}\right)^{3} \alpha_{m}^{3} \\
& \left(1+\bar{\sigma} / L_{1}\right)^{2} e^{-k_{\min } \gamma_{0} \bar{\sigma}}\left\|\tilde{u}^{s}\right\|_{H^{1 / 2}\left(\Gamma_{1}\right)},
\end{aligned}
$$

where $k_{\min }=k_{0} \min \left(n_{-}, n_{+}\right), k_{\max }=k_{0} \max \left(n_{-}, n_{+}\right), \gamma_{1}=e^{L_{2} \sqrt{\left|k_{\max }^{2}-k_{\min }^{2}\right|} / 2}$, $\alpha_{m}=\sqrt{1+\sigma^{2}}$, and $\hat{C}=\frac{\min \left(1, \sigma^{3}\right)}{2\left(1+\sigma^{2}\right)^{2} \max \left(1, k_{\max }^{2} d^{2}\right)}$.

Proof. The PML problem can be considered in $B_{2} / \bar{D}$ by regarding $D$ as an obstacle and enforcing the Dirichlet boundary condition $\left.\tilde{u}^{s}\right|_{\partial D}=\left.u^{s}\right|_{\partial D}$. Evidently, this theorem follows directly from Theorem 7.2 in [8].

Thanks to Theorem 2.2, $\tilde{u}^{s}$ converges to $u^{s}$ exponentially in $B_{1}$. Therefore, we only need to deal with $\tilde{u}^{s}$ in the bounded domain $B_{2}$ instead of $u^{s}$ in $\mathbb{R}^{2}$.

\section{$3 \quad$ Numerical mode matching method}

For the scattering problem formulated above, the NMM methods are applicable, since the structure is uniform in $x$ in three different segments corresponding to $x<-x_{0},-x_{0}<x<x_{0}$ and $x>x_{0}$, respectively. Since a PML is used in the NMM method, we define the three segments by $S_{1}=$ $\left\{(x, y) \mid-d_{1}-L_{1} / 2<x<-x_{0}\right\} \cap B_{2}, S_{2}=\left\{(x, y) \mid-x_{0}<x<x_{0}\right\} \cap B_{2}$ and $S_{3}=\left\{(x, y) \mid x_{0}<x<d_{1}+L_{1} / 2\right\} \cap B_{2}$. It is clear that $\varepsilon(x, y)=\varepsilon_{i}(y)$ in $S_{i}$ is independent of $x$, for $i=1,2,3$. Accordingly, $\Gamma_{2}$ (the boundary of $B_{2}$ ) is decomposed into three parts $\Gamma_{2}^{1}, \Gamma_{2}^{2}$ and $\Gamma_{2}^{3}$. In particular,

$$
\Gamma_{2}^{2}=\left\{\left(x, d_{2}+L / 2\right)|| x \mid<x_{0}\right\} \cup\left\{\left(x,-d_{2}-L / 2\right)|| x \mid<x_{0}\right\} .
$$


In the last several decades, many different NMM methods have been developed. These methods use different numerical methods to solve the eigenmodes in the uniform segments, and also use different techniques to impose the continuity conditions at the interfaces between the neighboring segments. Our NMM method is similar to the one presented in [22], and its basic steps are summarized below.

We consider segments $S_{1}$ and $S_{3}$ first. According to Eqs. (8, 10), $\tilde{u}^{s}$ in $S_{i}$ $(i=1,3)$ solves

$$
\begin{aligned}
& \nabla \cdot\left(A \nabla \tilde{u}^{s}\right)+k_{0}^{2} \alpha_{1}(x) \alpha_{2}(y) \varepsilon_{i}(y) \tilde{u}^{s}=0, \\
& \tilde{u}^{s}(x, 0+)=\tilde{u}^{s}(x, 0-), \quad \partial_{\tilde{y}} \tilde{u}^{s}(x, 0+)=\partial_{\tilde{y}} \tilde{u}^{s}(x, 0-), \\
& \tilde{u}^{s}\left(x, d_{2}+L_{2} / 2\right)=\tilde{u}^{s}\left(x,-d_{2}-L_{2} / 2\right)=0 .
\end{aligned}
$$

By the method of separation of variables, inserting $\tilde{u}^{s}(x, y)=\phi(y) \psi(x)$ into (12 14, we obtain the following eigenvalue problem for $\phi(y)$

$$
\begin{aligned}
& \frac{1}{\alpha_{2}} \frac{d}{d y}\left(\frac{1}{\alpha_{2}} \frac{d \phi}{d y}\right)+k_{0}^{2} \varepsilon_{i}(y) \phi(y)=\delta \phi, \\
& \phi(0+)=\phi(0-), \phi^{\prime}(0+)=\phi^{\prime}(0-), \\
& \phi\left(d_{2}+L_{2} / 2\right)=\phi\left(-d_{2}-L_{2} / 2\right)=0,
\end{aligned}
$$

and the associated equation for $\psi(x)$

$$
\frac{1}{\alpha_{1}} \frac{d}{d x}\left(\frac{1}{\alpha_{1}} \frac{d \psi}{d x}\right)+\delta \psi=0 .
$$

The above Sturm-Liouville eigenvalue problem (15, 17) for $\phi$ is not selfadjoint, thus $\delta$ is in general complex. Nevertheless, $\delta$ can be forced to the upper half-plane based on the following proposition.

Proposition 3.1. Under the same assumptions as Theorem 2.2, we have that for sufficiently large $\bar{\sigma}, \operatorname{Im}(\delta) \geq 0$.

Proof. See Proposition A.1 in Appendix A.

As in [29], we employ a pseudospectral method to find the numerical eigenmodes. Assuming $N$ eigenpairs $\left\{\delta_{j}, \phi_{j}(y)\right\}$ for $j=1, \ldots, N$, are obtained based on the $N$ collocation points $\left\{y^{j}\right\}_{j=1}^{N} \subset\left[-d_{2}-L_{2} / 2, d_{2}+L_{2} / 2\right]$, we approximate $\tilde{u}^{s}$ by

$$
\left.\tilde{u}^{s} \approx \sum_{j=1}^{N}\left[c_{j}^{(1)} e^{-i \sqrt{\delta_{j}}\left(\tilde{x}(x)-\tilde{x}\left(-x_{0}\right)\right)}+d_{j}^{(1)} e^{i \sqrt{\delta_{j}}\left(\tilde{x}(x)-\tilde{x}\left(-d_{1}-L_{1} / 2\right)\right)}\right] \phi_{j}^{\text {eq }} ;\right)^{\text {region }}(19)
$$


in $S_{1}$, and by

$$
\tilde{u}^{s} \approx \sum_{j=1}^{N}\left[c_{j}^{(3)} e^{-i \sqrt{\delta_{j}}\left(\tilde{x}(x)-\tilde{x}\left(d_{1}+L_{1} / 2\right)\right)}+d_{j}^{(3)} e^{i \sqrt{\delta_{j}}\left(\tilde{x}(x)-\tilde{x}\left(x_{0}\right)\right)}\right] \phi_{j}(\underline{y})^{\text {eq region }}(20)
$$

in $S_{3}$, where $\sqrt{\delta_{j}}$ is defined to be in the branch with $\operatorname{Im}\left(\sqrt{\delta_{j}}\right) \geq 0$ and hence with $\operatorname{Re}\left(\sqrt{\delta_{j}}\right) \geq 0$ according to Proposition 1 . Based on the zero Dirichlet boundary condition at $x= \pm\left(d_{1}+L_{1} / 2\right)$, we get

$$
\begin{aligned}
& d_{j}^{(1)}=-c_{j}^{(1)} e^{-i \sqrt{\delta_{j}}\left(\tilde{x}\left(-d_{1}-L_{1} / 2\right)-\tilde{x}\left(-x_{0}\right)\right)}, \\
& c_{j}^{(3)}=-d_{j}^{(3)} e^{i \sqrt{\delta_{j}}\left(\tilde{x}\left(d_{1}+L_{1} / 2\right)-\tilde{x}\left(-x_{0}\right)\right)} .
\end{aligned}
$$

Therefore,

$$
\begin{aligned}
& \left|d_{j}^{(1)}\right|=\left|c_{j}^{(1)}\right| e^{-\operatorname{Im}\left(\sqrt{\delta_{j}}\right)\left(L_{1} / 2-x_{0}+d_{1}\right)-\operatorname{Re}\left(\sqrt{\delta_{j}}\right) \int_{L_{1} / 2}^{L_{1} / 2+d_{1}} \sigma(t) d t} \approx 0, \\
& \left|c_{j}^{(3)}\right|=\left|d_{j}^{(3)}\right| e^{-\operatorname{Im}\left(\sqrt{\delta_{j}}\right)\left(L_{1} / 2-x_{0}+d_{1}\right)-\operatorname{Re}\left(\sqrt{\delta_{j}}\right) \int_{L_{1} / 2}^{L_{1} / 2+d_{1}} \sigma(t) d t} \approx 0,
\end{aligned}
$$

for sufficiently large $\sigma$ and $d_{1}$. Consequently, we can assume that there are no terms with coefficients $d_{j}^{(1)}$ and $c_{j}^{(3)}$ in Eqs. 19p and 20, respectively. Physically, this corresponds to the fact that $u^{s}$ should not contain incoming waves in the two exterior segments.

In segment $S_{2}$, we have

$$
\varepsilon_{2}(y)=\left\{\begin{array}{lc}
n_{+}^{2}, & y>y_{1} \\
\tilde{\varepsilon}(y), & y_{0}<y<y_{1}, \\
n_{-}^{2}, & y<y_{0} .
\end{array}\right.
$$

The method of separation of variables is not applicable to $\tilde{u}^{s}$, since it does not satisfy the homogeneous transmission conditions (3) at $y=y_{0}$ and $y=y_{1}$. Instead, we need to subtract from $u^{\text {tot }}$ a wave field $u_{2}^{\text {tot }}$ which solves the scattering problem for the same incident wave and a layered profile $\varepsilon(x, y)=$ $\varepsilon_{2}(y)$ in $\mathbb{R}^{2}$. We let $u_{2}^{\text {tot }}$ be the solution with the same $x$-dependence as the incident wave. More details are given Proposition A.2.

For $u_{2}^{s}=u^{\text {tot }}-u_{2}^{\text {tot }}$, we enforce the same zero Dirichlet boundary condition

$$
\tilde{u}_{2}^{s}=0, \quad \text { on } \Gamma_{2}^{2},
$$

where $\tilde{u}_{2}^{s}(x, y)=u_{2}^{s}(\tilde{x}(x), \tilde{y}(y))$. The method of separation of variables can be applied to $\tilde{u}_{2}^{s}$. Based on the same discretization points $\left\{y^{j}\right\}_{j=1}^{N}$, we obtain $N$ eigenpairs $\left\{\delta_{j}^{(2)}, \phi_{j}^{(2)}(y)\right\}_{j=1}^{N}$ in $S_{2}$, then

$$
\tilde{u}_{2}^{s} \approx \sum_{j=1}^{N}\left[c_{j}^{(2)} e^{-i \sqrt{\delta_{j}^{(2)}}\left(x-x_{0}\right)}+d_{j}^{(2)} e^{i \sqrt{\delta_{j}^{(2)}}\left(x+x_{0}\right)}\right] \phi_{j}^{(2)}(y) .^{\text {eq:region } 2}(25)
$$


On the two interfaces between $S_{2}$ and the other two segments $S_{1}$ and $S_{3}$, i.e. at $x= \pm x_{0}$, we have the transmission conditions

$$
\begin{aligned}
{\left[\tilde{u}_{s}\left( \pm x_{0}, y\right)-\tilde{u}_{s}^{2}\left( \pm x_{0}, y\right)\right] } & =\left[\tilde{f}\left( \pm x_{0}, y\right)\right], \\
{\left[\partial_{x} \tilde{u}_{s}\left( \pm x_{0}, y\right)-\partial_{x} \tilde{u}_{s}^{2}\left( \pm x_{0}, y\right)\right] } & =\left[\tilde{g}\left( \pm x_{0}, y\right)\right],
\end{aligned}
$$

where $\tilde{f}(x, y)=f(x, \tilde{y}(y)), \tilde{g}(x, y)=g(x, \tilde{y}(y))$, and

$$
f(x, y)=u_{2}^{\text {tot }}(x, y)-u_{0}^{\text {tot }}(x, y), \quad g(x, y)=\partial_{x} u_{2}^{\text {tot }}(x, y)-\partial_{x} u_{0}^{\text {tot }}(x, y) .
$$

Collocating (26) and (27) at $y=y^{j}$ for $j=1, \ldots, N$, and using Eqs. (19), (20) and 25), we obtain a linear system

$$
\boldsymbol{A}\left[\begin{array}{l}
\boldsymbol{c}^{(1)} \\
\boldsymbol{c}^{(2)} \\
\boldsymbol{d}^{(2)} \\
\boldsymbol{d}^{(3)}
\end{array}\right]=\boldsymbol{b}
$$

where $\boldsymbol{A}$ is a $4 N \times 4 N$ matrix, $\boldsymbol{b}$ is a $4 N \times 1$ matrix, $\boldsymbol{c}^{(i)}=\left[c_{1}^{(i)}, \ldots, c_{N}^{(i)}\right]^{T}$, etc. Solving the above system, we get $\tilde{u}^{s}$ in $S_{1}$ and $S_{3}$, and $\tilde{u}_{2}^{s}$ in $S_{2}$, thus $u^{\text {tot }}$ can be found in the physical domian $B_{1}$.

In the above, the NMM method is only presented for the case of a single inhomogeneous segment in a two-media layered background. It is straightforward to extend the NMM method to structures with multiple inhomogeneous segments that are uniform along the same direction. The NMM method can also be used to study scattering problems in the $H$ polarization (the only nonzero component of the magnetic field is its $z$ component) and problems involving perfect electrical conductor (PEC) or perfect magnetic conductor (PMC) scatterers.

\section{A Robin-type boundary condition}

As we mentioned in the introduction, the NMM method based on the zero Dirichlet condition (24) usualy works, but in some special circumstances, it exhibits a slow convergence and even a divergence, since $u_{2}^{s}$ may not be strictly outgoing. It should be pointed out that there is no contradiction with Theorem 2.2, since that theorem is about applying the PML to $u^{s}$, but the NMM method applies the PML to $u_{2}^{s}$ for the interior segment $S_{2}$.

In fact, it is easy to deduce that

$$
u_{2}^{s}=\left(R-R_{2} e^{-2 i \beta_{+} y_{1}}\right) e^{i\left(\alpha x+\beta_{+} y\right)}+u^{s}, \quad \text { for } y>y_{1}, \text { eq:us2:asym }\left(29^{\prime}\right)
$$




$$
u_{2}^{s}=\left(T-T_{2} e^{i \beta_{-} y_{0}-i \beta_{+} y_{1}}\right) e^{i\left(\alpha x-\beta_{-} y\right)}+u^{s}, \quad \text { for } y<y_{0},
$$

where $R$ and $R_{2}$ ( $T$ and $T_{2}$ ) are the reflection (transmission) coefficients in the reference solutions $u_{0}^{\text {tot }}$ and $u_{2}^{\text {tot }}$, respectively. Therefore, $u_{2}^{s}$ can be decomposed as a scattered wave field and an up-going plane wave with $y$ wavenumber $\beta_{+}$for $y>y_{1}$ or a down-going plane wave with $y$-wavenumber $\beta_{-}$for $y<y_{0}$. Consequently, only when $\beta_{+}$and $\beta_{-}$are sufficiently far away from zero, does $u_{2}^{s}$ attenuate in the PML. However, this is not ture for the following two cases:

a. For gracing incidences with $\theta$ close to $\pm \pi / 2, \beta_{+}$is close to 0 ;

b. For $n_{-}<n_{+}$and at the critical angles $\theta= \pm \arcsin \left(n_{-} / n_{+}\right)$for the onset of total internal reflection, $\beta_{-}=0$.

Notice that $\tilde{u}^{s} \approx 0$ at the exterior boundary of the PML, therefore

$$
\begin{aligned}
& \tilde{u}_{2}^{s} \approx\left(R-R_{2} e^{-2 i \beta_{+} y_{1}}\right) e^{i\left(\alpha x+\beta_{+} \tilde{y}\right)}, \quad \text { on } y=d_{2}+L_{2} / 2, \\
& \tilde{u}_{2}^{s} \approx\left(T-T_{2} e^{i \beta_{-} y_{0}-i \beta_{+} y_{1}}\right) e^{i\left(\alpha x-\beta_{-} \tilde{y}\right)}, \quad \text { on } y=-d_{2}-L_{2} / 2 .
\end{aligned}
$$

However, the NMM method is not compatible with the above inhomogeneous boundary conditions. To overcome this difficulty, the following result is needed.

Proposition 4.1. The scattered fields $u^{s}$ and $u_{2}^{s}$ satisfy

$$
\begin{gathered}
\partial_{y} u_{2}^{s}-i \beta_{+} u_{2}^{s}=\partial_{y} u^{s}-i \beta_{+} u^{s}, \quad \text { on } y=d_{2}+L_{2} / 2, \\
\partial_{y} u_{2}^{s}+i \beta_{-} u_{2}^{s}=\partial_{y} u^{s}+i \beta_{-} u^{s}, \quad \text { on } y=-d_{2}-L_{2} / 2 .
\end{gathered}
$$

Correspondingly,

$$
\begin{array}{r}
\left.\left.\left(A \nabla \tilde{u}_{2}^{s}\right) \cdot \nu-i \beta_{+} \tilde{u}_{2}^{s}=\left(A \nabla \tilde{u}^{s}\right) \cdot \nu-i \beta_{+} \tilde{u}^{s}, \quad \text { on } y=d_{2}+L_{2} / 2 \text { eq: tus: }: 33\right)_{1}\right) \\
\left(A \nabla \tilde{u}_{2}^{s}\right) \cdot \nu-i \beta_{-} \tilde{u}_{2}^{s}=\left(A \nabla \tilde{u}^{s}\right) \cdot \nu-i \beta_{-} \tilde{u}^{s}, \quad \text { on } y=-d_{2}-L_{2} / 2 .
\end{array}
$$

Proof. The proof is straightforward.

The above proposition suggests the following homogeneous Robin boundary conditions,

$$
\begin{aligned}
& \frac{1}{\alpha_{2}} \partial_{y} \tilde{u}_{2}^{s}-i \beta_{+} \tilde{u}_{2}^{s}=\left(A \nabla \tilde{u}_{2}^{s}\right) \cdot \nu-i \beta_{+} \tilde{u}_{2}^{s}=0, \quad \text { on } \quad y=d_{2}+\mathscr{L}: q 2 \text {, } \\
& \frac{1}{\alpha_{2}} \partial_{y} \tilde{u}_{2}^{s}+i \beta_{+} \tilde{u}_{2}^{s}=\left(A \nabla \tilde{u}_{2}^{s}\right) \cdot \nu-i \beta_{-} \tilde{u}_{2}^{s}=0, \quad \text { on } \quad y=-d_{2} \text { eq:cond:tus: } L_{2}^{2}{ }^{2}
\end{aligned}
$$


Based on the pseudospectral method [29] and the above boundary conditions, we can find the eigenmodes $\phi_{j}^{(2)}(1 \leq j \leq N)$, and expand $\tilde{u}_{2}^{s}$ in $S_{2}$ in these eigenmodes.

Although different boundary conditions are used on $\Gamma_{2}$, the following theorem ensures that $\tilde{u}^{s}$ still converges to $u^{s}$ exponentially.

Theorem 4.1. Under the same assumptions as Theorem 2.2, we have that for sufficiently large $\bar{\sigma}$, the PML problem (8), (9) equipped with the following hybrid Dirichlet-Robin boundary condition

$$
\left\{\begin{array}{l}
\tilde{u}^{s}=0, \\
\left(A \nabla \tilde{u}^{s}\right) \cdot \boldsymbol{\nu}=i W^{2} \tilde{u}^{s}, \quad \text { on } \quad \Gamma_{2} / \bar{\Gamma}
\end{array}\right.
$$

eq: general : cond

where $W \in L^{\infty}(\Gamma)$ is real-valued and $\Gamma \subset \Gamma_{2}$ is an open bounded set, has a unique solution $\tilde{u}^{s}$ in $H^{1}\left(B_{2}\right)$. Moreover, there exists a constant $C$, which depends only on $\left\|W^{2}\right\|_{L^{\infty}(\Gamma)}, \gamma_{0}, k_{\max } / k_{\min }$, and $L_{2} / L_{1}$, but independent of $n_{-}, n_{+}, L_{1}, L_{2}$, and $d$, such that

$$
\begin{aligned}
\left\|u^{s}-\tilde{u}^{s}\right\|_{H^{1}\left(B_{1}\right)} & \leq C\left(1+\hat{C}^{-1}\right) \gamma_{1}\left(1+k_{\min } L_{1}\right)^{3} \alpha_{m}^{3} \\
& \left(1+\bar{\sigma} / L_{1}\right)^{2} e^{-k_{\min } \gamma_{0} \bar{\sigma}}\left\|\tilde{u}^{s}\right\|_{H^{1 / 2}\left(\Gamma_{1}\right)} .
\end{aligned}
$$

Proof. This can be proved by the similar argument as the proof of Theorem 7.2 in [8]. We here only mention significant modifications. For consistency and simplicity, we will load the whole notations from [8] and will use them only in this proof so that $x=\left(x_{1}, x_{2}\right)$ now denotes a point but not a scalar, etc..

The PML equation in the PML layer (see Eqs. (5.1-5.3) in [8]) for the generalized boundary condition (37) should be revised to

$$
\begin{aligned}
& \nabla \cdot(A \nabla w)+\alpha_{1} \alpha_{2} k^{2} w=0, \quad \text { in } \quad \Omega_{\mathrm{PML}}=B_{2} \backslash \bar{B}_{1}, \\
& {[w]_{\Sigma}=\left[\frac{\partial w}{\partial x_{2}}\right]_{\Sigma}=0 \quad \text { on } \quad \Sigma \cap \Omega_{\mathrm{PML}}} \\
& w=0 \text { on } \Gamma_{1}, \quad w=q \text { on } \Gamma_{2} \backslash \bar{\Gamma}, \quad(A \nabla w) \cdot \nu-i W^{2} w=\stackrel{\text { eq }}{\text { : proof }} \text { : } \text { pmleq }^{2} \text { (41) }
\end{aligned}
$$

where $q \in H^{1 / 2}\left(\Gamma_{2} / \bar{\Gamma}\right)$ and $\tilde{q} \in H^{-1 / 2}(\Gamma)$. Then, the related sesquilinear form $c: H^{1}\left(\Omega_{\mathrm{PML}}\right) \times H^{1}\left(\Omega_{\mathrm{PML}}\right) \rightarrow \mathbb{C}$ becomes

$c(\varphi, \psi)=\int_{\Omega_{P M L}}\left(A \nabla \varphi \cdot \nabla \bar{\psi}-\alpha_{1} \alpha_{2} k^{2} \varphi \bar{\psi}\right) d x-i \int_{\Gamma} W^{2} \varphi \bar{\psi} d s, \forall \varphi, \psi \in H_{0 / \Gamma}^{1}\left(\Omega_{\mathrm{PML}}\right)$,

where $H_{0 / \Gamma}^{1}\left(\Omega_{\mathrm{PML}}\right):=\left\{v \in H^{1}\left(\Omega_{\mathrm{PML}}\right): v=0\right.$ on $\left.\Gamma_{1} \cup \Gamma_{2} / \bar{\Gamma}\right\}$. The weak formulation of $(39-41)$ is: Find $w \in H^{1}\left(\Omega_{\mathrm{PML}}\right)$ such that Eq. 41 is satisfied and that

$$
c(w, v)=\left.\langle\tilde{q}, v\rangle\right|_{\Gamma}, \quad \forall v \in H_{0 / \Gamma}^{1}\left(\Omega_{\mathrm{PML}}\right) . \text { eq:proof : weakform }(42)
$$


Correspondingly, the weighted $H^{1}$-norm is revised to

$$
\|\| \varphi \|_{H^{1}(\Omega)}=\left(\|\nabla \varphi\|_{L^{2}(\Omega)}^{2}+\|k \varphi\|_{L^{2}(\Omega)}^{2}+\|W \varphi\|_{\Gamma}^{2} /\left(1+\sigma^{2}\right)^{2}\right)^{1 / 2},
$$

and the equivalent norm on $H^{1}\left(\Omega_{\mathrm{PML}}\right)$ becomes

$$
\|\varphi\|_{*, \Omega_{\mathrm{PML}}}=\left(\|A \nabla \varphi\|_{L^{2}\left(\Omega_{\mathrm{PML}}\right)}^{2}+\left\|k \alpha_{1} \alpha_{2} \varphi\right\|_{L^{2}\left(\Omega_{\mathrm{PML}}\right)}^{2}+\|W \varphi\|_{\Gamma}^{2}\right)^{1 / 2} .
$$

Next, one sees that Lemma 5.1 in [8] still holds with the space $H_{0}^{1}$ replaced by $H_{0 / \Gamma}^{1}$. The proof relies on the following estimates

$$
\|\varphi\|_{L^{2}\left(\Omega_{1}\right)}^{2} \leq d_{1}^{2}\left\|\frac{\partial \varphi}{\partial x_{1}}\right\|_{L^{2}\left(\Omega_{1}\right)}^{2}, \quad\|\varphi\|_{L^{2}\left(\Omega_{2}\right)} \leq d_{2}^{2}\left\|\frac{\partial \varphi}{\partial x_{2}}\right\|^{2}
$$

which were proved by using $\varphi=0$ on $\Gamma_{2}$ in [8]. However, we remark that these two estimates still hold even when $\varphi \neq 0$ on $\Gamma_{2}$ since we always have $\varphi=0$ on $\Gamma_{1}$ for $\varphi \in H_{0 / \Gamma}^{1}\left(\Omega_{\mathrm{PML}}\right)$.

Thus, it is clear that Lemma 5.2 in [8] holds with the space of $\zeta$ replaced by "for any $\zeta \in H^{1}\left(\Omega_{\mathrm{PML}}\right)$ such that $\zeta=0$ on $\Gamma_{1}, \zeta=q$ on $\Gamma_{2} / \bar{\Gamma}$, and $A(\nabla \zeta) \cdot \nu-i W^{2} \zeta=\tilde{q}$ on $\Gamma$."

Next, Lemma 7.1 in [8] holds after one replaces $X(f)$ with

$$
\begin{aligned}
& \tilde{X}(f):=\left\{\zeta \in H^{1}\left(\Omega_{\mathrm{PML}}\right): \zeta=0 \text { on } \Gamma_{1}, \zeta=\mathbb{E}(f) \text { on } \Gamma_{2} / \bar{\Gamma},\right. \\
&\left.(A \nabla \zeta) \cdot \nu-i W^{2} \zeta=\left(A \nabla_{x} \mathbb{E}(f)\right) \cdot \nu-i W^{2} \mathbb{E}(f) \text { on } \Gamma\right\} .
\end{aligned}
$$

Here, we will have

$$
\begin{aligned}
\inf _{\zeta \in \tilde{X}}\|\zeta\|_{*, \Omega_{\mathrm{PML}}} \leq C\left(1+k_{1} L_{1}\right) \alpha_{m}^{2}( & \|\mathbb{E}(f)\|_{H^{1 / 2}\left(\Gamma_{2} / \bar{\Gamma}\right)} \\
& \left.+\left\|\left(A \nabla_{x} \mathbb{E}(f)\right) \cdot \nu-i W^{2} \mathbb{E}(f)\right\|_{H^{-1 / 2}}(\Gamma)\right),
\end{aligned}
$$

where $C$ now depends on the norm $\left\|W^{2}\right\|_{L^{2}\left(\Gamma_{2}\right)}$ considering the modified norm $\|\cdot\|_{*, \Omega_{\mathrm{PML}}}$. Since $\mathbb{E}(f)$ is smooth on $\Gamma$,

$$
\left(A \nabla_{x} \mathbb{E}(f)\right) \cdot \nu-i W^{2} \mathbb{E}(f) \in L^{2}(\Gamma) \cap L^{\infty}(\Gamma),
$$

so that

$$
\left\|(A \nabla \mathbb{E}(f)) \cdot \nu-i W^{2} \mathbb{E}(f)\right\|_{H^{-1 / 2}(\Gamma)} \leq C\|\mathbb{E}(f)\|_{W^{1, \infty}(\Gamma)},
$$

and hence Lemma 7.1 in [8] follows which proves the theorem. 
If we define $W$ in $\Gamma_{2}^{2}$ by

$$
W(x, y)=\left\{\begin{array}{lc}
\sqrt{\beta_{+}}, & \text {on } y=d_{2}+L_{2} / 2, \\
\sqrt{\beta_{-}}, & \text {on } y=-d_{2}-L_{2} / 2,
\end{array}\right.
$$

then Theorem 3 is applicable to our scattering problem. Consequently, with the hybrid Dirichlet-Robin condition (37), $\tilde{u}^{s}$ still exponentially converges to $u^{s}$ in the physical domain $B_{1}$.

Theorems 2.2 and 3.1 are established for PMLs with constant and equal $\sigma_{1}$ and $\sigma_{2}$. In practice, we may set $\sigma_{1}(x)$ and $\sigma_{2}(y)$ as continuous functions to increase flexibility. For example, we may choose

$$
\sigma_{l}(t)=\sigma\left(\frac{t-L_{l} / 2}{d_{l}}\right)^{m}, \quad \text { in } L_{l} / 2<|t|<L_{l} / 2+d_{l}^{\text {eq: }} \text { : pml : setup }(43)
$$

for a positive constant $\sigma$ and an integer $m \geq 0$, where $m=0$ corresponds to the constant case.

\section{Cylindrical incident waves}

The NMM methods are typically implemented for plane incident waves. For other incident waves, such as point or line sources and Gaussian beams, the NMM methods may be used with a Fourier transform that rewrites the incident wave as a superposition of plane waves. This approach is not very efficient, since it is necessary to solve the problem for many different incident plane waves. Alternatively, we can try to find a reference solution for the given non-plane incident wave in each uniform segment. This task is nontrivial for the interior segment corresponding to the inhomogeneity. In the following, we present an efficient method for computing the reference solutions when the incident wave is a cylindrical wave generated by a line source.

The incident cylindrical wave is $u^{\text {inc }}=\frac{i}{4} H_{0}^{(1)}\left(k_{0} n_{+} \rho(x, y)\right)$ corresponding to a line source at $\left(x^{*}, y^{*}\right) \in \mathbb{R}_{+}^{2} / \bar{D}$, where $\rho(x, y)=\sqrt{\left(x-x^{*}\right)^{2}+\left(y-y^{*}\right)^{2}}$. The governing Helmholtz equation becomes

$$
\Delta u^{\mathrm{tot}}+k_{0}^{2} \varepsilon(x, y) u^{\mathrm{tot}}=-\delta\left(x-x^{*}\right) \delta\left(y-y^{*}\right) .
$$

Considering the location of the source, we have the following three cases:

(a) If $\left|x^{*}\right|<x_{0}$, we set $u_{0}^{\text {tot }} \equiv 0$ and find a nonzero $u_{2}^{\text {tot }}$;

(b) If $\left|x^{*}\right|>x_{0}$ and $y^{*}>y_{1}$, we set $u_{2}^{\text {tot }} \equiv 0$ and find a nonzero $u_{0}^{\text {tot }}$; 
(c) If $\left|x^{*}\right|=x_{0}$, then we have to find nonzero $u_{0}^{\text {tot }}$ and $u_{2}^{\text {tot }}$.

We consider the typical case (a), where $u_{2}^{\text {tot }}$ must be computed in segment $S_{2}$. The NMM method requires $u_{2}^{\text {tot }}$ and its $x$-derivative at $x= \pm x_{0}$ to evaluate $\tilde{f}$ and $\tilde{g}$ in Eqs. 26 and 27 .

Following the one-dimensional profile $\varepsilon_{2}(y)$ given in $(23), \mathbb{R}^{2}$ can be split into three layers $y<y_{0}, y_{0}<y<y_{1}$, and $y>y_{1}$. The wave field

$$
u^{s}= \begin{cases}u_{2}^{\text {tot }}-u^{\text {inc }}, & \text { in } y>y_{1} \\ u_{2}^{\text {tot }}, & \text { otherwise }\end{cases}
$$

is outgoing as $y \rightarrow \pm \infty$. Using the same PML as before and applying the technique of separation of variables to $\tilde{u}^{s}$, we obtain the following eigenvalue problem

$$
\begin{aligned}
& \frac{1}{\alpha_{1}} \frac{d}{d x}\left(\frac{1}{\alpha_{1}} \frac{d \psi}{d x}\right)=\delta \psi, \\
& \psi\left(-L_{1} / 2-d_{1}\right)=\psi\left(L_{1} / 2+d_{1}\right)=0,
\end{aligned}
$$

and its associated equation

$$
\begin{aligned}
\frac{1}{\alpha_{2}} \frac{d}{d y}\left(\frac{1}{\alpha_{2}} \frac{d \phi}{d y}\right)+\left(k_{0}^{2} \varepsilon_{2}(y)+\delta\right) \phi & =0 \\
\phi\left(-L_{2} / 2-d_{2}\right)=\phi\left(L_{2} / 2+d_{2}\right) & =0 .
\end{aligned}
$$

Different from the main step of the NMM method, the separation of variables here leads to an eigenvalue problem for $\psi$ (a function of $x$ ), instead of $\phi$ which is not continuous at $y=y_{1}$.

The eigenvalue problem for $\psi$ can be solved by a pesudospectral method as in [29]. If $M$ numerical eigenpairs $\left\{\delta_{j}, \psi_{j}(x)\right\}_{j=1}^{M}$ are obtained corresponding to the collocation points $\left\{x_{j}\right\}_{j=1}^{M} \subset\left[-L_{1} / 2-d_{1}, L_{1} / 2+d_{1}\right]$, we approximate $\tilde{u}^{s}$ by

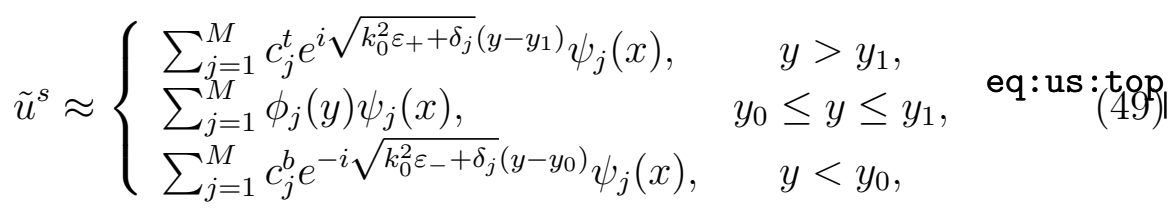

where the square roots have nonnegative imaginary parts. Notice that only outgoing waves are retained in the top and bottom layers.

The functions $\phi_{j}(y)$ satisfy

$$
\frac{d^{2} \phi_{j}}{d y^{2}}+\left[k_{0}^{2} \tilde{\varepsilon}(y)+\delta_{j}\right] \phi_{j}=0 .
$$


Since $\tilde{u}^{s}$ satisfies the transmission condition at $y=y_{0}$, we have

$$
\phi_{j}\left(y_{0}\right)=c_{j}^{b}, \quad \phi_{j}^{\prime}\left(y_{0}+\right)=-c_{j}^{b} i \sqrt{k_{0}^{2} \varepsilon_{-}+\delta_{j}} .
$$

Therefore, we enforce the following Robin boundary condition

$$
\phi_{j}^{\prime}\left(y_{0}+\right)=-i \sqrt{k_{0}^{2} \varepsilon_{-}+\delta_{j}} \phi_{j}\left(y_{0}\right)
$$

At $y=y_{1}$, we can find the coefficients $\left\{c_{j}^{\mathrm{ps}}, d_{j}^{\mathrm{ps}}\right\}_{j=1}^{N}$ such that

$$
\begin{aligned}
& \sum_{j=1}^{N} c_{j}^{\mathrm{ps}} \psi_{j}(x)=\frac{i}{4} H_{0}^{(1)}\left(k_{0} n_{+} \rho\left(\tilde{x}(x), y_{1}\right)\right) \\
& \sum_{j=1}^{N} d_{j}^{\mathrm{ps}} \psi_{j}(x)=\left.\frac{d}{d y} \frac{i}{4} H_{0}^{(1)}\left(k_{0} n_{+} \rho(\tilde{x}(x), y)\right)\right|_{y=y_{1}}
\end{aligned}
$$

are exactly satisfied at the collocation points $\left\{x_{j}\right\}_{j=1}^{N}$. Since $u^{\text {tot }}$ satisfies the transmission condition on $y=y_{1}$, we have

$$
c_{j}^{t}+c_{j}^{\mathrm{ps}}=\phi_{j}\left(y_{1}\right), \quad i c_{j}^{t} \sqrt{k_{0}^{2} \varepsilon_{+}+\delta_{j}}+d_{j}^{\mathrm{ps}}=\phi_{j}^{\prime}\left(y_{1}-\right) .
$$

Eliminating $c_{j}^{t}$, the above yields the following Robin boundary condition,

$$
\phi_{j}^{\prime}\left(y_{1}-\right)-i \sqrt{k_{0}^{2} \varepsilon_{+}+\delta_{j}} \phi_{j}\left(y_{1}\right)=d_{j}^{\mathrm{ps}}-i \sqrt{k_{0}^{2} \varepsilon_{+}+\delta_{j}} c_{j}^{\mathrm{ps}} \cdot \text { eq }: \mathrm{RBC}:(50 \mathrm{t})
$$

As shown in Proposition A.2, the boundary value problem (50), (51) and (54) has a unique solution. Using a pesudospectral method, we solve this boundary value problem and obtain $\phi_{j}(y)$ at the collocation points $\left\{y^{j}\right\}_{j=1}^{N} \cap$ $\left[y_{0}, y_{1}\right]$. Finally, since $c_{j}^{b}=\phi_{j}\left(y_{0}\right)$ and $c_{j}^{t}=\phi_{j}\left(y_{1}\right)-c_{j}^{\mathrm{ps}}$, we have $\tilde{u}^{s}$ and $u_{2}^{\text {tot }}$ in $B_{2}$. The other two cases (b) and (c) are similar; we omit the details here.

\section{$6 \quad$ Numerical examples}

In this section, we carry out several numerical experiments to exhibit the performance of our NMM method. In all examples, the physical domain is chosen to be $(-2.5,2.5) \times(-2.5,2.5)$, and the free-space wavenumber $k_{0}=$ $2 \pi / \lambda$ with wavelength $\lambda=1.13$.

Example 1. In the first example, the background two-layer medium is separated by interface $y=0$, with $\varepsilon(x, y)=4$ in the top, and $\varepsilon(x, y)=1$ in the bottom. The inhomgeneity filled in domain $D=(-0.5,0.5) \times(-1,1)$ is 
the same as the medium in the top, so that it functions as a local perturbation to the interface $y=0$; see the dashed lines in Fig. 2. The PML-BIE method recently developed in 21] is applicable to this problem and is used to validate our NMM method. Using 1000 points to discretize the interface in the PMLBIE method, we obtain a reference solution $u_{\text {ref }}^{\text {tot }}$. To quantify the accuracy of the NMM method, we define the following relative error,

$$
e_{\mathrm{rel}}=\frac{\max _{(x, y) \in S}\left|u_{\mathrm{ref}}^{\mathrm{tot}}(x, y)-u_{\mathrm{NMM}}^{\mathrm{tot}}(x, y)\right|}{\max _{(x, y) \in S}\left|u_{\mathrm{ref}}^{\mathrm{tot}}(x, y)\right|} .
$$

Notice that $e_{\text {rel }}$ compares the numerical solution by the NMM method with the reference solution on the set $S=\{(x, y) \mid x= \pm 0.5, y= \pm 2.5,-1,0\}$. The choice of $S$ is typical, since it contains all corners on the interfaces and some points at the interior boundary of the PML.

First, we validate the Robin-type boundary condition. We choose $\sigma=70$, $d=0.05$, and $m=0$ to set up the PML. For both $E$ and $H$ polarizations, we choose $N=950$ eigenmodes in each segment, and compute $e_{\text {rel }}$ for incident angle $\theta$ varying in $[0, \pi / 2)$. The results are shown in Figs. 1(a) and 1(b). It is clear that in the vicinity of the critical angle $\theta=\pi / 6$, where total internal reflection first occurs, or $\theta=\pi / 2$, which gives horizontally propagating incident plane waves, the Robin boundary condition produces a much smaller $e_{\text {rel }}$ and significantly outperforms the Dirichlet boundary condition.

At both the critical incident angle $\theta=\pi / 6$ and the normal incidence with $\theta=0$, we study the relation between $e_{\text {rel }}$ and the PML thickness $d$ for a fixed $\sigma=70$. The numerical results are shown in Figs. 1(c) and 1(d), where both axes are scaled logarithmically. When $d$ is small, we expect that the error is dominated by the truncation of the PML. The results in Figs. 11(c) and 1 (d) indicate that $e_{\text {rel }}$ initially decays exponentially as $d$ is increased. This behavior is expected from Theorem 3.1.

Finally, we compare the numerical solutions by the NMM and PML-BIE methods for two types of incident waves: a plane wave with the critical incident angle $\theta=\pi / 6$, and a cylindrical wave excited by a source at $(0.2,1)$. The results are shown in Fig. 2. For each case, the PML-BIE solution is shown on the left and the NMM solution is shown on the right. Clearly, the solutions obtained by the two numerical methods are nearly indistinguishable from each other.

Example 2. The dielectric function $\varepsilon(x, y)$ is profiled by Fig. 3(c), where $\varepsilon(x, y)$ is 4 in the top layer, 1 in the bottom layer, and $(1.5+y)^{2}$ in the inhomogeneity $D=(-0.5,0.5) \times(-0.5,0.5)$.

Since $\varepsilon(x, y)$ is variable in $D$, the PML-BIE method is no longer applicable. We use the NMM method to find the total field $u^{\text {tot }}$ for the $E$ 
(a)

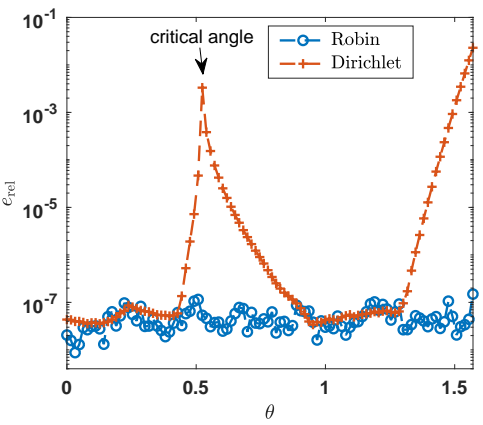

(c)

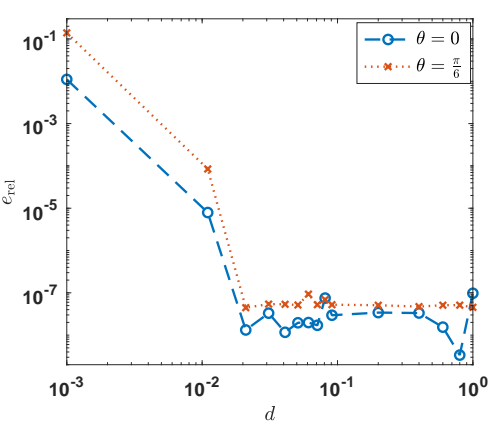

(b)

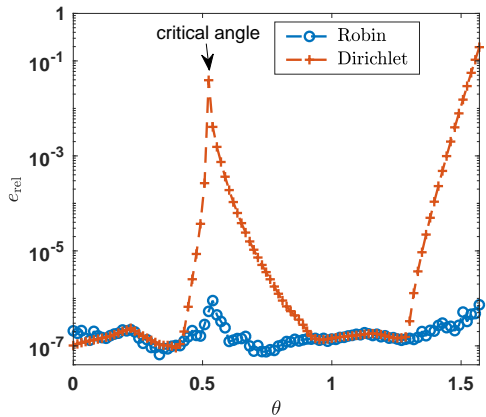

(d)

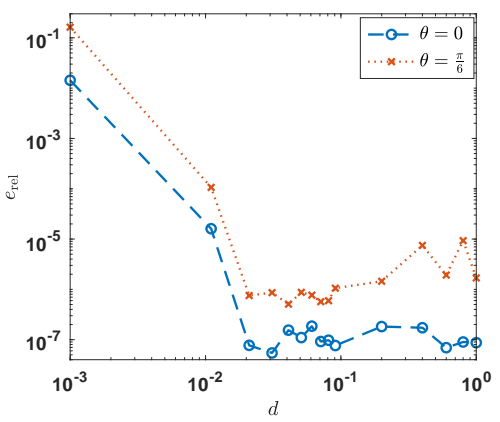

Figure 1: Example 1: (a) and (b): Relative error versus incident angle $\theta$ using Robin and Dirichlet boundary conditions on $\Gamma_{2}^{2}$, for $N=950, \sigma=70$, $d=0.05$, and $m=0$. (a) $E$ polarization; (b) $H$ polarization. (c) and (d): Relative error versus PML thickness $d$ at $\theta=0$ and $\theta=\pi / 6$, for $N=950$, $m=0$ and $\sigma=70$ : (c) $E$ polarization; (d) $H$ polarization.

polarization and for two different incident waves: a plane wave at the critical incident angle $\theta=\pi / 6$, and a cylindrical wave excited by a source at $(0.2,1)$.

For these two incident waves, using $N=534$ eigenmodes in each segment, and using $m=0, \sigma=70$ and $d=1$ to set up the PML, we obtain two numerical solutions, relatively, as shown in Figs. 3(a) and 3(b). Using the above two numerical solutions as reference solutions, relatively, we compute $e_{\text {rel }}$ defined in Eq. (55), but for $S=\{(x, y) \mid x= \pm 0.5, y= \pm 0.5, \pm 2.5\}$ for numerical solutions with values of $d$ less than 1 , for the two incident waves, relatively; see Fig. 3(d). As before, when $d$ is small, the relative error decays exponentially, since it is dominated by the truncation of the PML.

Example 3. The dielectric function $\varepsilon(x, y)$ is profiled by Fig. 4 (c), where two $y$-dependent inhomogeneities are embedded in the background medium with three layers. Here, $\varepsilon(x, y)$ is $4,2.25$ and 1 in the top, inner, and bottom 
(a)
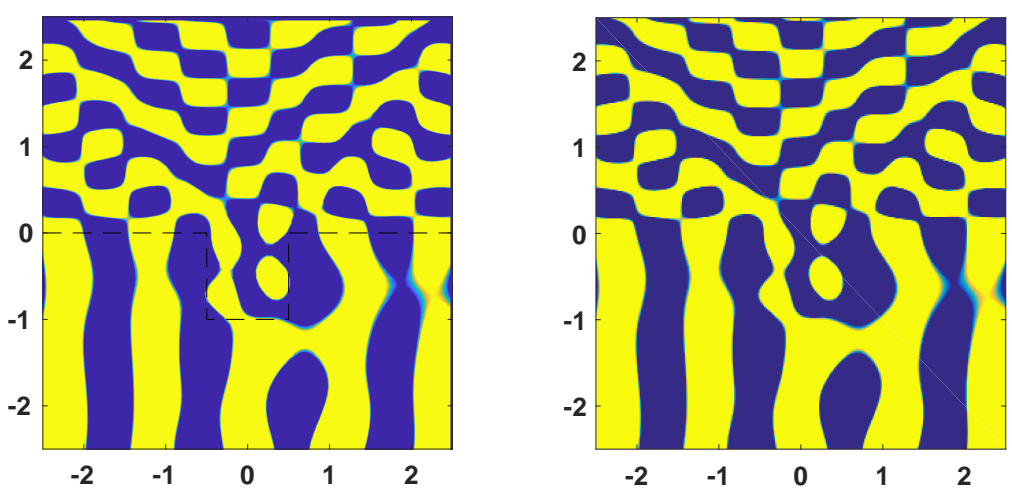

(b)
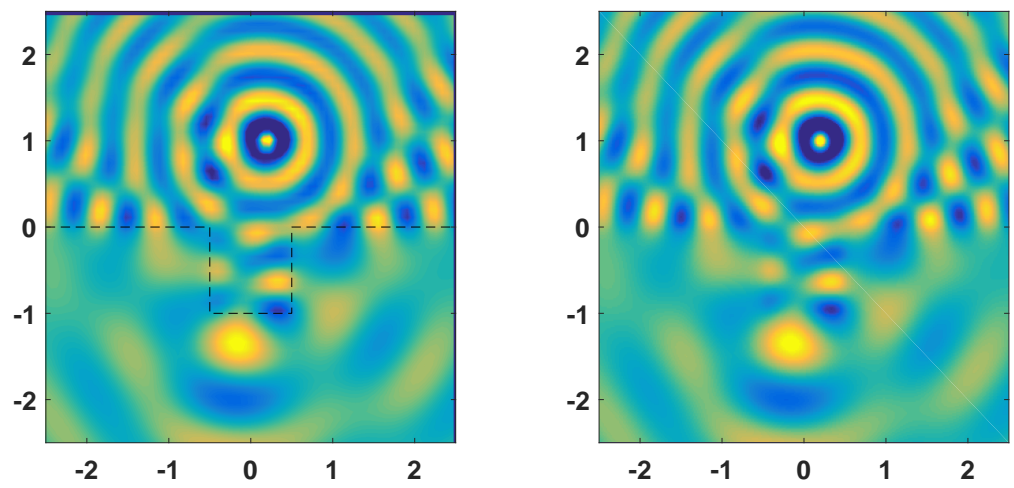

Figure 2: Example 1: Numerical solutions of a scattering problem in $E$ polarization: (a) plane wave at critical incident angle $\theta=\pi / 6$; (b) cylindrical wave for a source at $(0.2,1)$. For both (a) and (b), the reference solution by the PML-BIE method is shown on the left and the numerical solution by the NMM method is shown on the right.

layers, respectively, and in the two inhomogeneities,

$$
\varepsilon(x, y)= \begin{cases}\left(1+\sin ^{2}(\pi y / 2)\right)^{2}, & (x, y) \in D_{1}=(-1.5,-0.5) \times(-1,1) \\ \left(1+\cos ^{2}(\pi y / 2)\right)^{2}, & (x, y) \in D_{2}=(0.5,1.5) \times(-1,1)\end{cases}
$$

Using $N=633$ eigenmodes in each segment, and using $m=0, \sigma=70$, and $d=1$ to set up the PML, we calculate the total field $u^{\text {tot }}$ for the $E$ polarization and for two different incident waves: a plane wave with the critical incident angle $\theta=\pi / 6$, and a cylindrical wave excited by a source at $(-0.7,1.2)$. The results are shown in Figs. $4(\mathrm{a})$ and $4(\mathrm{~b})$. 


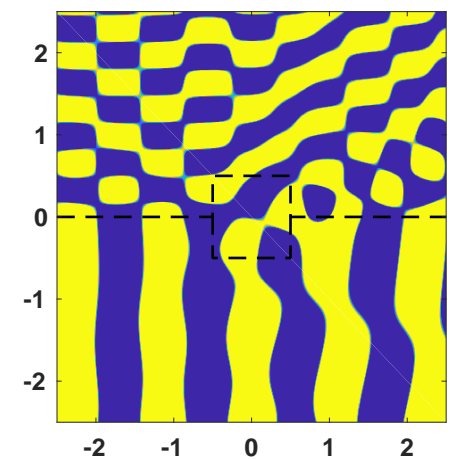

(a)

(c)

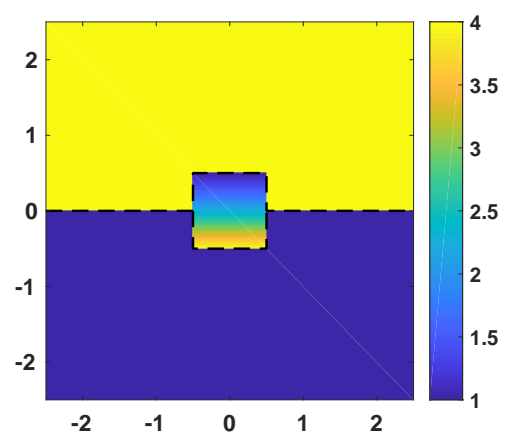

(b)

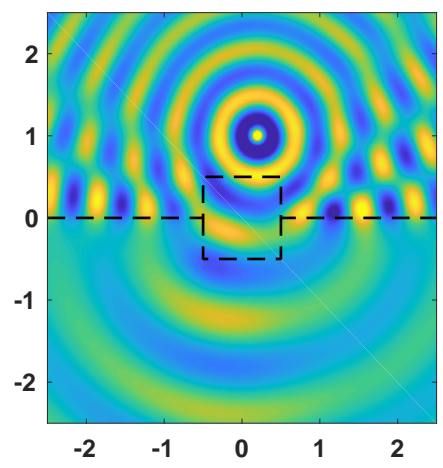

(d)

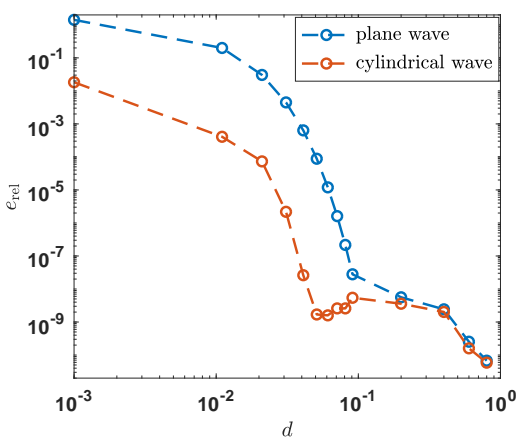

Figure 3: Example 2: Numerical solutions of a scattering problem in the $E$ polarization: (a) plane wave at critical incident angle $\theta=\pi / 6$; b) cylindrical wave for a source at $(0.2,1)$; (c) profile of the dielectric function $\varepsilon(x, y)$; (d) relative error $e_{\text {rel }}$ versus PML thickness $d$. In (a) and (b), we take $N=534$, $m=0, \sigma=70$ and $d=1$.

\section{Conclusion}

The NMM methods are widely used in engineering applications for simulating propagation and scattering of linear electromagnetic, acoustic and elastic waves. These methods are restricted to special structures, but are more efficient than the standard numerical methods when they are applicable, since no discretization is needed for one spatial variable. In this paper, a new NMM method is developed to overcome a limitation of existing NMM methods due to the existence of a non-propagating and non-decaying wave field component. A Robin-type boundary condition is used to ensure that the wave field component with zero or near-zero transverse wavenumber is not reflected by a truncated PML. A theoretical foundation of the new NMM is established by a theorem which reveals the exponential convergence of the PML solution with the hybrid Dirichlet-Robin boundary conditions. In ad- 


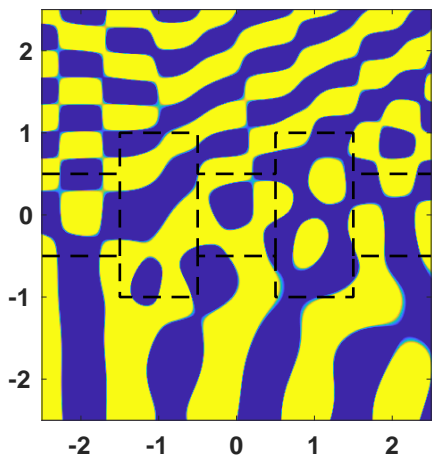

(a)

(c)

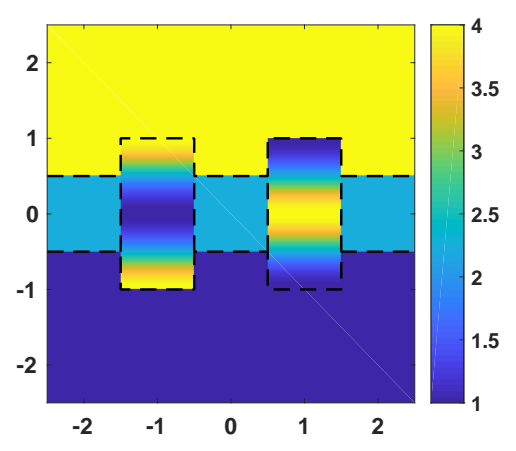

Figure 4: Example 3: Numerical solutions of a scattering problem in the $E$ polarization: (a) plane wave at critical incident angle $\theta=\pi / 6$; (b) cylindrical wave for a source at $(-0.7,1.2)$; (c) profile of the dielectric function $\varepsilon(x, y)$. Other parameters: $N=633, m=0, \sigma=70$, and $d=1$.

dition, for scattering problems with cylindrical incident waves, we developed a fast method to compute reference solutions needed in the NMM methods. Numerical examples are presented to validate the NMM method and illustrate its performance.

We have implemented the new NMM method for two-dimensional structures with one or more inhomogeneities, for electromagnetic waves in $E$ and $H$ polarizations, and for both plane and cylindrical incident waves. The NMM methods are also applicable to three-dimensional (3D) rotationally symmetric structures that are piecewise uniform in the radial variable [15, 22. There is also a related method for more general 3D structures without the rotational symmetry [28, 27]. The techniques developed in this paper, namely, the Robin-type boundary condition for terminating the PML and the fast method for computing reference solutions for cylindrical incident waves, should also be useful in these NMM and related methods for 3D structures. 


\section{Appendix A Proof of Propositions}

Proposition A.1. Under the same assumptions as Theorem 2.2, we consider the eigenvalue problem (15,17) with $\varepsilon_{i}$ replaced by

$$
\varepsilon_{\mathrm{gen}}(y)=\left\{\begin{array}{lc}
\varepsilon_{+}, & y>y_{1} \\
\varepsilon_{-}, & y<y_{0} \\
\varepsilon_{\mathrm{PHY}}(y), & y_{0} \leq y \leq y_{1}
\end{array}\right.
$$

where $\varepsilon_{\mathrm{PHY}}(y)$ can be any piecewise smooth and positive function. Then, one and only one of the following two cases occurs:

(a) There exist $\sigma^{0}>0$ and $d^{0}>0$ such that if $\sigma>\sigma^{0}$ or if $d>d^{0}$, then $\operatorname{Im}(\delta) \geq 0$ for any eigenpair $\{\phi, \delta\}$ that solves 15,17 );

(b) For a fixed $d>0(\sigma>0)$, there exist a sequence of $\left\{\sigma^{n}\right\}_{n=1}^{\infty}\left(\left\{d^{n}\right\}_{n=1}^{\infty}\right.$, respectively) that approaches infinity as $n \rightarrow \infty$ such that there exists a sequence of associated eigenpairs $\left\{\phi^{n}, \delta^{n}\right\}$ satisfying $\operatorname{Im}\left(\delta^{n}\right)<0$.

If case (b) holds, then $\operatorname{Im}\left(\delta^{n}\right) \rightarrow 0$ as $n \rightarrow \infty$ and for sufficiently large $n$,

$$
\operatorname{Re}\left(\delta^{n}\right) \in\left[\max \left(k_{0}^{2} \varepsilon_{+}, k_{0}^{2} \varepsilon_{-}\right), \max \left(k_{0}^{2} \varepsilon_{\mathrm{PHY}}\right)\right) .
$$

Proof. It is clear that if case (a) does not hold, then case (b) must hold. We now prove $\operatorname{Im}\left(\delta^{\mathrm{n}}\right) \rightarrow 0$ as $n \rightarrow \infty$. Integrating 15 with $\bar{\phi}$ on $\left[-L_{2} / 2, L_{2} / 2\right]$ and using integration by parts yield

$$
\begin{aligned}
\delta \int_{-L_{2} / 2}^{L_{2} / 2}|\phi|^{2} d y= & -\int_{-L_{2} / 2}^{L_{2} / 2}\left|\phi^{\prime}(y)\right|^{2} d y+k_{0}^{2} \int_{-L_{2} / 2}^{L_{2} / 2} \varepsilon_{\mathrm{PHY}}|\phi|^{2} d y \\
& +\left.\left(\frac{d \phi}{d y} \bar{\phi}\right)\right|_{-L_{2} / 2} ^{L_{2} / 2} \text { eq: } \operatorname{de} t
\end{aligned}
$$

In $\mathrm{PML}_{y}=\left(-L_{2} / 2-d,-L_{2} / 2\right) \cup\left(L_{2} / 2, L_{2} / 2+d\right), \phi(y)$ has the following general solution form,

$$
\phi(y)=c_{ \pm}^{1} e^{ \pm i k_{ \pm}^{*}\left(\tilde{y} \mp L_{2} / 2\right)}+c_{ \pm}^{2} e^{\mp i k_{ \pm}^{*}\left(\tilde{y} \mp L_{2} / 2\right)}, \quad \text { in } \mathrm{PML}_{y} \cap \mathbb{R}_{ \pm},
$$

where $k_{ \pm}^{*}=\sqrt{k_{0}^{2} \varepsilon_{ \pm}-\delta}$ with $\operatorname{Re}\left(k_{ \pm}^{*}\right) \geq 0$. The homogeneous Dirichlet boundary condition at $y= \pm\left(L_{2} / 2+d\right)$ implies that

$$
c_{ \pm}^{2}=-c_{ \pm}^{1} e^{ \pm i 2 k_{ \pm}^{*} d(1+i \sigma)},
$$

so that, by a straightforward calculation, one obtains

$$
\left.\left(\frac{d \phi}{d y} \bar{\phi}\right)\right|_{-L_{2} / 2} ^{L_{2} / 2}=\sum_{l= \pm}\left|c_{l}^{1}\right|^{2}\left[\left(1-e^{-4 d\left(\operatorname{Im}\left(k_{l}^{*}\right)+\operatorname{Re}\left(k_{l}^{*}\right) \sigma\right)}\right) i k_{l}^{*}\right.
$$




$$
\left.-2 k_{l}^{*} e^{-2 d\left(\operatorname{Im}\left(k_{l}^{*}\right)+\operatorname{Re}\left(k_{l}^{*}\right) \sigma\right)} \sin \left(2 d\left(\operatorname{Re}\left(k_{l}^{*}\right)-\operatorname{Im}\left(k_{l}^{*}\right) \sigma\right)\right)\right] \text { eq:est: imag }
$$

If $\operatorname{Im}(\delta)<0$, then $\operatorname{Im}\left(k_{ \pm}^{*}\right)>0$. Next, we show that $\operatorname{Re}\left(k_{ \pm}^{*, n}\right) \rightarrow 0$ as $n \rightarrow \infty$, where $k_{ \pm}^{*, n}=\sqrt{k_{0}^{2} \varepsilon_{ \pm}-\delta^{n}}$ with nonnegative real part. Otherwise, suppose we have a subsequence $\left\{n_{k}\right\}_{k=1}^{\infty}$ such that $\operatorname{Re}\left(k_{ \pm}^{*, n_{k}}\right) \rightarrow c_{ \pm}^{0}>0$ as $n_{k} \rightarrow \infty$. Considering the imaginary part of (57),

$$
\left.\operatorname{Im}\left(\frac{d \phi^{n_{k}}}{d y} \bar{\phi}^{n_{k}}\right)\right|_{-L_{2} / 2} ^{L_{2} / 2} \rightarrow\left|c_{+}^{1}\right|^{2} c_{+}^{0}+\left|c_{-}^{1}\right|^{2} c_{-}^{0}>0,
$$

which implies that $\operatorname{Im}\left(\delta^{n_{k}}\right)>0$. Considering the real part of (57),

$$
\left.\operatorname{Re}\left(\frac{d \phi}{d y} \bar{\phi}\right)\right|_{-L_{2} / 2} ^{L_{2} / 2} \leq \sum_{l= \pm}\left|c_{l}^{1}\right|^{2}\left[-\left(1-e^{-4 d\left(\operatorname{Im}\left(k_{l}^{*}\right)+\operatorname{Re}\left(k_{l}^{*}\right) \sigma\right)}\right) \operatorname{Im}\left(k_{l}^{*}\right)+2 \operatorname{Re}\left(k_{l}^{*}\right)\right] .
$$

For sufficiently large $n, \operatorname{Re}\left(k_{l}^{*, n}\right)$ can be arbitrarily small such that

$$
\left.\operatorname{Re}\left(\frac{d \phi^{n}}{d y} \bar{\phi}^{n}\right)\right|_{-L_{2} / 2} ^{L_{2} / 2} \leq 0
$$

Therefore, considering the real part of (56),

$$
\operatorname{Re}\left(\delta^{n}\right)<k_{0}^{2} \max \left(\varepsilon_{\mathrm{PHY}}\right),
$$

since $\frac{d}{d y} \phi^{n}(y) \neq 0$ in $\left[-L_{2} / 2, L_{2} / 2\right]$. On the other hand,

$$
\operatorname{Re}(\delta)=k_{0}^{2} \varepsilon_{ \pm}-\operatorname{Re}\left(k_{ \pm}^{*}\right)^{2}+\operatorname{Im}\left(k_{ \pm}^{*}\right)^{2} \geq k_{0}^{2} \varepsilon_{ \pm}-\operatorname{Re}\left(k_{ \pm}^{*}\right)^{2} .
$$

so that $\operatorname{Re}\left(\delta^{n}\right) \geq k_{0}^{2} \varepsilon_{ \pm}$and that

$$
\operatorname{Im}\left(k_{ \pm}^{*, n}\right)<k_{0}^{2} \max \left(\varepsilon_{P H Y}\right)-k_{0}^{2} \varepsilon_{ \pm}+\operatorname{Re}\left(k_{ \pm}^{*, n}\right)^{2},
$$

Consequently, as $n \rightarrow \infty$, we have $\operatorname{Im}\left(\delta^{n}\right)=-2 \operatorname{Im}\left(k_{ \pm}^{*, n}\right) \operatorname{Re}\left(k_{ \pm}^{*, n}\right) \rightarrow 0$.

Proposition A.2. If $\tilde{\varepsilon}(y)$ is smooth on $\left[y_{0}, y_{1}\right]$, the following field

$$
u_{2}^{\text {tot }}=e^{-i \beta_{+} y_{1}} \begin{cases}e^{i \alpha x}\left(e^{\left.-i \beta_{+}\left(y-y_{1}\right)\right)}+R_{2} e^{i \beta_{+}\left(y-y_{1}\right)}\right) & \text { if } y \geq y_{1} \dot{\text { eqq }} \text { : closed:form } \\ e^{i \alpha x} f(y), & \text { if } y_{0} y \dot{<} y_{1}, \\ T_{2} e^{i\left(\alpha x-\beta_{-}\left(y-y_{0}\right)\right)}, & \text { if } y \leq y_{0} .\end{cases}
$$

solves the scattering problem (2) and (3) with $\varepsilon(x, y)=\varepsilon_{2}(y)$ in $\mathbb{R}^{2}$, where $\beta_{-}$was defined in (6), the unknown function $f \in C^{2}\left[y_{0}, y_{1}\right]$ is uniquely determined by the following boundary value problem

$$
f^{\prime \prime}+\left(k_{0}^{2} \varepsilon_{2}-\alpha^{2}\right) f=0,
$$




$$
\begin{aligned}
& f^{\prime}\left(y_{0}+\right)=-i \beta_{-} f\left(y_{0}\right), \\
& f^{\prime}\left(y_{1}-\right)=i \beta_{+}\left(f\left(y_{1}\right)-2\right),
\end{aligned}
$$

and

$$
R_{2}=f\left(y_{1}\right)-1, \quad T_{2}=f\left(y_{0}\right) .
$$

Proof. The verification that $u_{2}^{\text {tot }}$ defined in $(58)$ is indeed a solution is straightforward. One only needs to prove that the boundary value problem (59, 61) has a unique solution. By the standard ODE theory, one needs to show that equation (59) with the following homogeneous Robin boundary conditions

$$
f^{\prime}\left(y_{0}+\right)=-i \beta_{-} f\left(y_{0}\right), \quad f^{\prime}\left(y_{1}-\right)=i \beta_{+} f\left(y_{1}\right)
$$

has only the trivial solution $f=0$. To show this, integrating 59 with $\bar{f}$ on $\left[y_{0}, y_{1}\right]$ yields, by integration by parts,

$$
\int_{y_{0}}^{y_{1}} f^{\prime} \bar{f}^{\prime} d y-\left(k_{0}^{2} \tilde{\varepsilon}-\alpha^{2}\right) f \bar{f} d y-\left(i \beta_{+} f\left(y_{1}\right) \bar{f}\left(y_{1}\right)+i \beta_{-} f\left(y_{0}\right) \bar{f}\left(y_{0}\right)\right)=0 .
$$

Considering the imaginary part of the left-hand side, we have

$$
\beta_{+}\left|f\left(y_{1}\right)\right|^{2}+\beta_{-}\left|f\left(y_{0}\right)\right|^{2}=0,
$$

so that $f\left(y_{1}\right)=0$ since $\beta_{+}>0$. Notice that $\beta_{-}$could be zero when total internal reflection occurs. Then, $f^{\prime}\left(y_{1}-\right)=i \beta_{+} f\left(y_{1}\right)=0$ indicates that $f=0$ on $\left[y_{0}, y_{1}\right]$ which completes the proof.

Remark A.1. $u_{2}^{\text {tot }}$ in (58) plus any guided mode or any surface mode, if there exists, still solves the scattering problem.

\section{References}

[1] G. Bao, G. Hu, and T. Yin. Time-harmonic acoustic scattering from locally perturbed half-planes. submitted, 2018.

[2] J.-P. Berenger. A perfectly matched layer for the absorption of electromagnetic waves. J. Comput. Phys., 114(2):185 - 200, 1994.

[3] P. Bienstman and R. Baets. Optical modelling of photonic crystals and vcsels using eigenmode expansion and perfectly matched layers. Opt. Quant. Electron., 33:327-341, 2016. 
[4] P. Bienstman, H. Derudder, R. Baets, F. Olyslager, and D. De Zutter. Analysis of cylindrical waveguide discontinuities using vectorial eigenmodes and perfectly matched layers. IEEE Trans. Microw. Theory Tech., 49:349-354, 2001.

[5] L.C. Botten, M.S. Craig, and R.C. McPhedran. Highly conducting lamellar diffraction gratins. Optica Acta, 28:1103-1106, 1981.

[6] O. P. Bruno, M. Lyon, C. Pérez-Arancibia, and C. Turc. Windowed Green function method for layered-media scattering. SIAM Journal on Applied Mathematics, 76(5):1871-1898, 2016.

[7] W. Cai. Algorithmic issues for electromagnetic scattering in layered media: Green's functions, current basis, and fast solver. Advances in Computational Mathematics, 16(2):157-174, 2002.

[8] Z. Chen and W. Zheng. Convergence of the uniaxial perfectly matched layer method for time-harmonic scattering problems in two-layered media. SIAM J. Numer. Analy., 48:2158-2185, 2010.

[9] W. C. Chew. Waves and fields in inhomogeneous media. IEEE PRESS, New York, 1995.

[10] Y.-P. Chiou, W.-L. Yeh, and N.-Y. Shih. Analysis of highly conducting lamellar gratings with multidomain pseudospectral method. J. Lightwave Technol., 27:5151-5159, 2009.

[11] H. Derudder, De Zutter D., and F. Olyslager. Analysis of waveguide discontinuities using perfectly matched layers. Electron. Lett., 34:2138$2140,1998$.

[12] G. Granet. Reformulation of the lamellar grating problem through the concept of adaptive spatial resolution. J. Opt. Soc. Am. A, 16:2510$2516,1999$.

[13] G. Granet and B. Guizal. Efficient implementation of the coupled-wave method for mettalic lamellar gratings in tm polarization. J. Opt. Soc. Am. A, 13:1019-1023, 1996.

[14] P. Ho and Y. Y. Lu. A mode preserving perfectly matched layer for optical waveguides. IEEE Photon. Tech. Lett., 15(9):1234-1236, 2003.

[15] Z. Hu, J. Lin, Y. Y. Lu, and S.-H. Oh. Fast vertical mode expansion method for the simulation of extraordinary terahertz field enhancement in an annular nanogap. J. Opt. Soc. Am. B, 35:30-38, 2018. 
[16] K. Knop. Rigorous diffraction theory for transmission phase gratings with deep rectangular grooves. J. Opt. Soc. Am., 68:1206-1210, 1978.

[17] J. Lai, L. Greengard, and M. OŃeil. A new hybrid integral representation for frequency domain scattering in layered media. Appl. Comput. Harmon. Anal., in press, 2016.

[18] P. Lalane and G. M. Morris. Highly improved convergence of the coupled-wave method for tm polarization. J. Opt. Soc. Am. A, 13:779$784,1996$.

[19] L. Li. A modal analysis of lamellar diffraction gratings in conical mountings. J. Mod. Opt., 40:553-573, 1993.

[20] L. Li. Use of fourier series in the analysis of discontinuous periodic structures. J. Opt. Soc. Am. A, 13:1870-1876, 1996.

[21] W. Lu, Y. Y. Lu, and J. Qian. Perfectly matched layer boundary integral equation method for wave scattering in a layered medium. SIAM J. Appl. Math., 78(1):246-265, 2018.

[22] X. Lu, H. Shi, and Y. Y. Lu. Vertical mode expansion method for transmission of light through a circular hole in a slab. J. Opt. Soc. Am. A, 31:293-300, 2014.

[23] P. Monk. Finite Element Methods for Maxwell's Equations. Oxford University Press, 2003.

[24] R. H. Morf. Exponentially convergent and numerically efficient solution of Maxwell's equations for lamellar gratings. J. Opt. Soc. Am. A, 12:1043-1056, 1995.

[25] G. F. Roach and B. Zhang. The limiting-amplitude principle for the wave propagation problem with two unbounded media. Math. Proc. Cambridge Philos. Soc., 112:207-223, 1992.

[26] P. Sheng, R. S. Stepleman, and P. N. Sanda. Exact eigenfunctions for square wave gratings - application to diffraction and surface-plasmon calculations. Phys. Rev. B, 26:2907-2916, 1982.

[27] H. Shi, X. Lu, and Y. Y. Lu. Vertical mode expansion method for numerical modeling of biperiodic structures. J. Opt. Soc. Am. A, 33:836$844,2016$. 
[28] H. Shi and Y. Y. Lu. Efficient vertical mode expansion method for scattering by arbitrary layered cylindrical structures. Opt. Expr., 23:1461814629, 2015.

[29] D. Song, L. Yuan, and Y. Y. Lu. Fourier-matching pseudospectral modal method for diffraction gratings. J. Opt. Soc. Am. A, 28:613-620, 2011. 